\title{
Using The Tenets Of Problem Based Learning In The Development Of Small Group Experiences In The Rehabilitative Environment
}

Genevieve Pinto Zipp PT, EdD, Seton Hall University, USA

Ashlesha Shah, PTA, BS Ex. Sci., MSHS, Graduate Student, Seton Hall University, USA

\begin{abstract}
Purpose. Professional health science educational programs have utilized numerous learning strategies including journals, practice patterns, problem-based learning (PBL), case-studies, and hypothesis-oriented algorithm for clinicians (HOAC) (Shepard et al., 2002). While, these learning strategies have found a place in the health science educational curriculums they have not been utilized in the clinic with patients. This essay provides a brief overview on the tenets of small group work associated with PBL and offers insight into how small groups may be used in the clinic as a teaching strategy. Importance. While this essay does not offer data to support the use of small group work as a learning technique for the promotion of patient problem solving it does provide a medium for the exchange of ideas which may lead to future research in this area.
\end{abstract}

Keywords: Small groups, problem based learning, educational strategy, learning strategy

\section{INTRODUCTION}

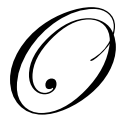

ne teaching method that has been used widely in health profession education is that of problem based learning (PBL). Proponents of PBL suggest that it encourages the promotion of effective clinical reasoning and problem solving. In PBL the focus is on the use of small group work where students collaborate to reach a common goal (Saarinen-Rahiika \& Binkley, 1998). Conversely, critics of PBL suggest that clinical expertise is related to the extent of the professional's knowledge base. The de-emphasis on content in the PBL teaching method may hamper the process association with problem solving and this should be used selectively within a highly structured curricular framework (Jefferson, 2001). The promotion of learning via the use of small group work experiences similar to those used in PBL has been supported by several theories, particularly Gage and Berliner's information-processing model (McGinty, 2000) and Colbert's metacognition theory (McGinty, 2000). With today's health care practices undergoing constant change health care professionals must make increasingly complex clinical decisions daily and patients must actively participate in the problem solving process in order to function safely in their environments. Educators within health professional programs must not only use teaching strategies that promote active learning in their students, they must explore how these strategies can be used by their students when working with patients for the promotion of learning. The purpose of this essay is to review the tenets of small group work used in the PBL teaching method, discuss its usage in promoting patient problem solving skills in clinical practice through the use of small group motor experiences and offer insight on how an educator might instruct a professional student on the use of this strategy through a case experience.

\section{CRITERIA AND COMPONENTS OF SMALL GROUP WORK}

The success of the small group teaching method in the academic environment is dependent on many factors, including: group size; the instructor's role in the group; the identification of objectives; discussion of 
acquired information and clinical application; self-study in between sessions and finally evaluation of self, peer and tutor at end of each session (Saarinen-Rahiika \& Binkley, 1998).

Saarinen-Rahiika \& Binkley (1998) suggest that an effective group size usually consists of 6-9 students and a faculty member who meets 2-3 times per week for up to 2-3 hours per session. To date, however, no consistent findings have been provided in the literature regarding the optimal group size (Jefferson, 2001). Overall, the group size should be small enough so that each member can benefit and actively engage in the discussion or task at hand.

Once the small group is formed the goals of PBL session are presented to the group by the instructor via the case scenario. The scenario provides initial direction to the students for navigating through the vast amount of information available. The instructor's role is to assist each member's ability to identify their role in the group and assist them to remain focused on the goals set forth by the scenario. The student's role is to critically review and analyze the information available in order to gain knowledge that would enable them to effectively address the goals set forth in the scenario. In a clinical scenario, the objective is to provide a context for learning, activate prior knowledge and motivate students (Saarinen-Rahiika \& Binkley, 1998). Most importantly, clinical scenarios with PBL facilitate discussion among students as they contemplate on different therapeutic interventions for the particular patient (Saarinen-Rahiika \& Binkley, 1998). The students are both active listeners as well as active learners because they are reacting intellectually to the other students' ideas or opinions. The instructor directs the discussions to help students reach the most appropriate intervention(s) for the scenario.

In contrast, during a traditional lecture approach the teacher provides all (majority) the information and students are passive learners. Critical thinking or analysis of the information given occurs on an individual basis for each student and may occur over time. This passive learning experience presents many problems when students are at clinical affiliations where they are faced with real-world problems and the difficulty of problem solving as well as critical thinking (Saarinen-Rahiika \& Binkley, 1998).

Clearly, small group work is a more student-directed teaching approach where the teacher is the tutor or mentor whose goal is to encourage students to critically think. Using this teaching strategy the faculty member becomes a facilitator of learning, by fostering self-directed learning in students and assisting students in the longitudinal integration of material (Saarinen-Rahiika \& Binkley, 1998). The instructor creates boundaries and a safe environment for exchange of ideas among students. He or she should ask questions that challenge students in the group to examine issues with multiple views and students should take positions and defend them especially in discussions as mentioned later (McGinty, 2000).

As Brookfield states (p.48), “...learning, and teaching are passionate, emotional activities", therefore, perhaps learning can be enhanced in small group work when subject matter presents a dilemma or especially provokes emotion like a controversial issue for instance. Discussions create cognitive conflict that motivates students to maintain a balance or equilibrium (McGinty, 2000). Students in small groups also develop group interaction skills and knowledge becomes that which they construct by talking together and reaching agreement (McGinty, 2000).

\section{THEORETICAL BASIS}

The information-processing model best explains how information presented to students in small groups moves from working memory to long term memory (McGinty, 2000). This stored information can then be classified as declarative and procedural knowledge but the discussions that are sparked in small groups, especially when the case method is used; give meaning from experience therefore, enhances better retention (McGinty, 2000). This model is similar to Schmidt's (1975) schema theory in motor learning which states that the key to successful future performance of a skill is the amount of variability in practice (Magill,284). In other words, students develop their own framework or schemata from the representation of the information presented and discussed in small groups in order to retain information and/or alter schemata when new information is added to memory. Simultaneously, pattern recognition is facilitated by PBL for students and patients so they can solve new problems based on relationships or patterns of observed previous behavior (Perry, 1999). What is learned in small groups is retained for 
longer period of time and a sort of guide is carried with each student when it is necessary to apply that information to the real world (McGinty, 2000).

Although some opponents of small group work claim that it is time consuming for faculty and promotes lower levels of content-specific knowledge, students overall in small groups present better retention over longer periods of time and spend less time in researching and studying as they become more competent information seekers (Saarinen-Rahiika \& Binkley, 1998).

\section{USE OF THE SMALL GROUP PBL STRATEGY IN THE REHABILITATIVE ENVIRONMENT AS TEACHING AND LEARNING TOOL}

Transfer of learning and the ability to apply small group work clinically is dependent on how close the learning task is to reality (McGinty, 2000). As physical therapists, the use of the small group PBL strategy in patient treatments may provide an environment for promoting problem solving, decision making skills, and clinical reasoning. The following are two case examples of how small group sessions which are used in rehabilitative departments can be enhanced using the premises associated with PBL in an attempt to promote patient problem solving skills. In this environment group sessions can include 5-6 patients with impairments resulting from a cerebral vascular accident. The small group can consist of one physical therapist, one therapy aide and the 5-6 patients. They can take place weekly for one 60 minutes session. The focus of these weekly group sessions can center on the performance of functional activities that promote dynamic and static balance abilities and ambulation skills using a PBL approach to learning.

Small Group 1 PBL: For example, when using the functional task of volleyball as the task practiced during the group, static and dynamic balance is a major focus. Patients can stand up either with/ without an assistive device to maintain upright balance while hitting the volleyball in all directions and keeping the volleyball from hitting the ground on either side of the net. As the mediator, the PT regulates the game, keeps score, provides rest intervals, and provides physical assistance only if necessary thus, enabling the patients to problem solve and learn to think on their own with reference to where or how to hit the volleyball to be successful at the game while maintaining their balance. Afterwards, the PT can facilitate a discussion amongst the group by asking them how they felt emotionally and physically during and after the activity.

Following the task performance and the discussion period the PT may ask questions addressing common problems they may encounter in their home to see if they can transfer what they have learned from the group situation to their home environment. For example, a question may ask, "what would you do to maintain standing balance if you had to reach for a cup or plate in the kitchen cupboard that was too high or too low; what things should you do or not do to promote safety?"

Small Group 2 PBL: Another clinical application of a small group PBL session could focus on working on safe transfers and bed mobility. For example, first patients in a group of four could perform particular transfers such as stand-pivot from a wheelchair to bed and vice versa with a therapist and an aide. Then the PT could facilitate discussion by asking each patient if they thought the transfer was completed safely and correctly. After the patient responds the question can be directed to the rest of the group for their comment on that patient's performance. After which the entire group can be asked to discuss other ways the task could have been done and finally to demonstrate it. Again this small group PBL experience encourages active patient learning and problem solving. If needed, the PT can offer alternate ways to perform the task and then ask the group to explain why this way maybe the safest. Finally, each group member can be asked to perform the task on that day, one week later in the therapy department and at their bedside to make certain they have learned the task and can transfer the performance to another environment.

\section{CONCLUSION}

While the use of small groups in promoting effective clinical reasoning and problem solving in the academic environment has been addressed in the PBL literature, it usage as a teaching and learning strategy in the clinical arena with patients has not been addressed. This essay is an attempt to discuss how the tenets associated 
with small group PBL can be used in attempt to foster patient problem solving and reasoning. Although this paper does not provide data to support the efficacy of small group PBL in clinical practice it does offer insight into how a small group could be arranged to potentially promote patient problem solving. Health science faculty can use the cases as a learning tool for students. The cases presented can serve as a framework to assist students in understanding how one uses a small group PBL format to promote active learning in the patient population. These cases may provide a vehicle for the development of future cases.

In future research the role of small group PBL for the promotion of clinical reasoning and patient problem solving should be addressed as it may provide an alternate avenue to meet the needs of our patients in the ever changing health care arena. Using a task analysis to assess the benefit of small group programming for the promotion of clinical reasoning and patient problem solving can provide an objective measurement of change. By determining the actual number of error producing responses compared to errorless responses made across sessions we can obtain a better understanding of the participants abilities to effectively problem solve the task requested. Also assessing the change across sessions with regard to reaction time, movement time and response time for each specific task may provide a window into the patient's problem solving strategies. Patients demonstrating a shorter reaction time across small group sessions would support the notion that the small group experience fostered their reasoning and problem solving skills thereby enabling them to react more efficiently.

Further research is needed on: optimum group size; potential differences associated with learning for various learning styles; gender differences; effectiveness when used in combination with other teaching methods; and reliability and validity of this teaching strategy in both the academic and clinical areas. However, the small group PBL strategy presented offers a unique way to promote teaching and learning in both health science students and the patients they teach.

\section{AUTHOR INFORMATION}

Dr. Pinto Zipp is Associate Professor and Chair of the Department of Graduate Programs in Health Sciences at Seton Hall University. She received her EdD from Teachers College Columbia University in 1996. She currently teaches management of neuromuscular problems in the Doctor of Physical Therapy program. Her research interests focus on, a) effects of performing dual tasks on walking performance and postural sway in children and adults, and b) curriculum design issues including the use of mind mapping and video based cases in professional education for the promotion of clinical decision making skills.

Ms. Ashley Parikh is a PTA working at the Texas Medical Center in Houston, TX. She completed her Master of Science in Health Science in the Department of Graduate Programs in Health Sciences at Seton Hall University in 2006. Her research interest focuses on evidence based practice in the health sciences.

\section{REFERENCES}

1. Binkley, M. J., \& Saarinen-Rahiika, H.(1998). Problem-Based Learning in Physical Therapy: A Review of the Literature and Overview of the McMaster University Experience. Physical Therapy, 78, 195-207.

2. Brookfield, S. D. (1990). The Skillful Teacher. San Francisco: Jossey-Bass Publishers. (Original work published 1990).

3. Jefferson, J. R., PT, MSc (2001). Problem-Based Learning and the Promotion of Problem Solving: Choices for Physical Therapy Curricula. Journal of Physical Therapy Education, 15 (1), 26-31.

4. McGinty, S. M. (2000). Case-Method Teaching: An Overview of the Pedagogy and Rationale for Its Use in Physical Therapy Education. Journal of Physical Therapy Education, 14(1), 48-51.

5. Magill, R. A. (2001). Motor Learning: Concepts and Principles (Sixth ed.). Boston: McGraw-Hill. (Original work published 1980).

6. Perry, S. B., MS, PT, NCS (1999). Strategies for Teaching Clinical Decision-Making Skills in Neurologic Content: Perspective from the Field. Neurology Report, 23 (4), 170-177. 